\title{
Suizidhilfe ist Teil der ärztlichen Aufgaben die Zeit ist reif für eine Anpassung der SAMW-Richtlinien
}

\author{
Die SAMW hat «Probleme bei der Durchführung von ärztlicher Suizidhilfe» diagnos- \\ tiziert und sich hierzu auch in der Schweizerischen Ärztezeitung geäussert. Nach \\ Ansicht der Autorin liegen die Probleme eher bei den SAMW-Richtlinien, denn sie \\ suggerierten, Suizidhilfe widerspreche dem ärztlichen Ethos, was nicht zutreffe. Frei- \\ todhilfe solle auch chronisch Kranken und anderen Leidenden offenstehen.
}

\section{Marion Schafroth \\ Vorstandsmitglied EXIT}

Lesen Sie zu diesem Thema auch das «Zu guter Letzt» auf Seite 1242 .
Korrespondenz: Dr. med. Marion Schafroth Vorstandsmitglied EXIT Mühlezelgstrasse 45 CH-8047 Zürich

marion.schafroth[at]gmail.com
Der Titel dieses Beitrags mag für gewisse Kolleginnen und Kollegen provokativ sein. Als lebenserfahrene Ärztin, FMH-Mitglied und Vorstandsmitglied der Sterbehilfeorganisation EXIT habe ich ihn absichtlich so formuliert. Seit zwei Jahren trage ich bei dieser Organisation die Verantwortung für das Ressort Freitodbegleitung.

Meine Meinungsäusserung ist eine wohlüberlegte Reaktion auf eine Stellungnahme der Zentralen Ethikkommission (ZEK) der Schweizerischen Akademie der Medizinischen Wissenschaften (SAMW) vom 20.1.2011. Sie wurde unter dem Titel «Probleme bei der Durchführung von ärztlicher Suizidhilfe» in der Schweizerischen Ärztezeitung Nr. 11 vom 14. März 2012 veröffentlicht.
Dieser skeptisch-ablehnenden Haltung der ZEK stelle ich entschieden eine andere Sicht der Dinge gegenüber:

- Geburt - Leben - Sterben: In allen Lebensphasen ist es höchstes ärztliches Ziel, den bestmöglichen Beitrag zum «guten Gelingen» zu leisten. Die Sorge dafür umfasst Prävention, Therapie und, wo diese nicht mehr möglich ist, Symptombekämpfung bzw. Leidenslinderung - alle diese Tätigkeiten sind immer begleitet von Fürsorge und menschlicher Zuwendung. Die Respektierung des Selbstbestimmungsrechts von urteilsfähigen und gutinformierten Patienten und Patientinnen darf, ja muss dabei heute als selbstverständlich gelten.

«In allen Lebensphasen ist es höchstes ärztliches Ziel, den bestmöglichen Beitrag zum «guten Gelingen`zu leisten.»

In diesem Artikel behauptet die ZEK, dass in Einzelfällen eine «nicht vertretbare Praxis der ärztlichen Suizidhilfe bestehe, und zwar teilweise mit, teils aber auch ohne Beteiligung einer Sterbehilfeorganisation». Sie verweist auf die SAMW-Richtlinien «Zur Betreuung von Patientinnen und Patienten am Lebensende» und warnt davor, dass deren Missachtung eine Verletzung des ärztlichen Standesrechts darstelle. Sie fordert eine Diskussion darüber «wie Missbräuche verhindert werden können und Ausweitungserscheinungen beurteilt werden sollten».

Mit ihren Äusserungen erweckt die ZEK fälschlicherweise den Eindruck, Sterbehilfeorganisationen und Ärzte mit der Bereitschaft zur Rezeptierung eines tödlichen Medikamentes verhielten sich öfter inkorrekt, und suggeriert, Suizidhilfe laufe dem ärztlichen Ethos zuwider.
- Hilfe zum Suizid ist nach sorgfältiger Prüfung bei Patienten mit schwerem Leiden und autonom entstandenem Sterbewunsch eine ethisch gerechtfertigte ärztliche Handlung - und dies explizit nicht nur im Falle unmittelbar zum Tod führender Krankheiten, sondern auch bei unheilbar chronischem schwerem Leiden oder schwerer Behinderung.

- Die Furcht vor der im Einzelfall manchmal schwierigen Auseinandersetzung zwischen den beiden Polen «Recht auf Freiheit, Menschenwürde und Privatsphäre» (bis zum Suizid) und dem «Recht auf Fürsorge» darf nicht dazu führen, dass Suizidhilfe der Einfachheit halber als dem ärztlichen Auftrag zuwiderlaufend abgelehnt wird. Wer, wenn nicht Ärztinnen und Ärzte, sollten besser dazu befähigt sein, die individuelle 


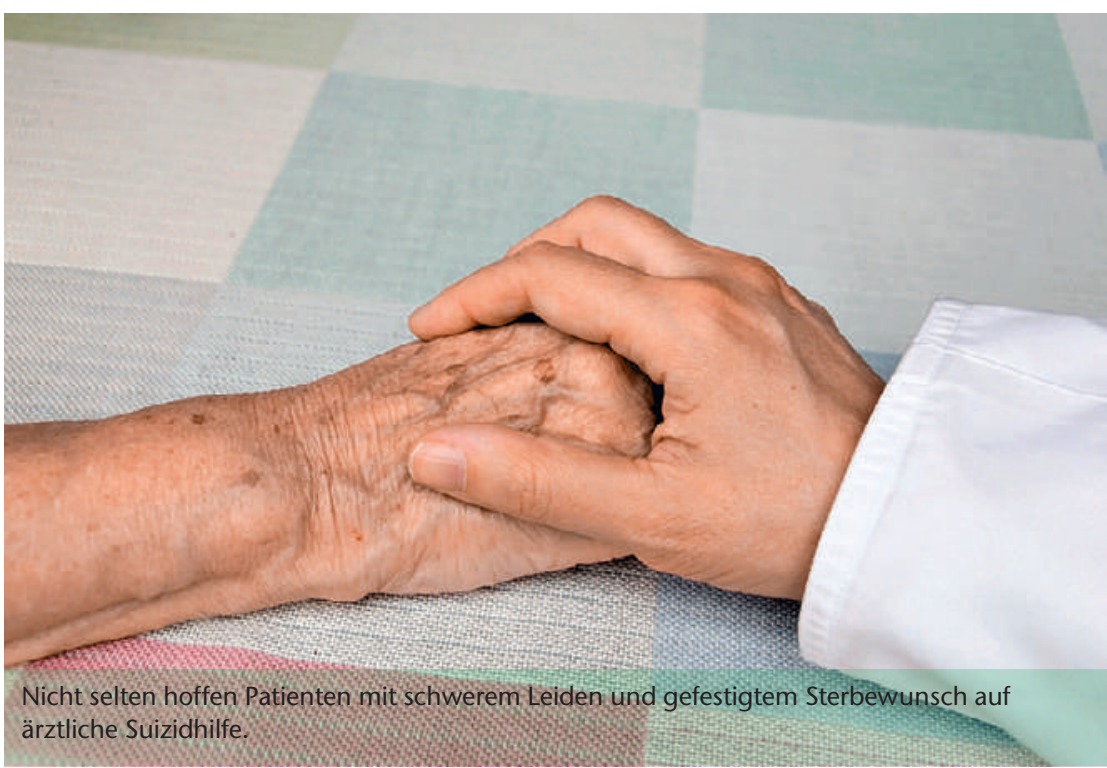

Krankheitssituation und die Urteilsfähigkeit verschiedenster Menschen in ihrer ganz individuellen Lebenssituation zu beurteilen und gegebenenfalls auf freiwilliger Basis das Rezept für ein Erlösung bringendes Medikament auszustellen?

- Im Jahre 2011 kam der Bundesrat zum Schluss, keine Verschärfung der gesetzlichen Bestimmungen über die Freitodhilfe anzustreben. Er deklarierte offiziell, dass ihm die Selbstbestimmung am Lebensende wichtig sei. Justizministerin Simonetta Sommaruga hat zudem öffentlich erklärt, Sterbehilfegesellschaften wie EXIT leisteten einen Beitrag zur Suizidprävention.

- Die vorausgegangene breite Vernehmlassung hatte klar ergeben, dass die Selbstbestimmung am Lebensende, wie in Bundesverfassung und der Europäischen Menschenrechtskonvention (EMRK) garantiert, zu respektieren sei, dass die heutige gesetzliche Regelung genüge, um etwaige Missbräuche zu verhindern, und dass die ärztliche Freitodhilfe nicht nur unmittelbar vom Tod bedrohten Patienten, sondern auch chronisch Kranken und anderen Leidenden offenstehen soll. -
Die von der ZEK geforderte breite Diskussion hat längst stattgefunden.

- Die Möglichkeit des ärztlich rezeptierten und liebevoll begleiteten Suizids ist von der Bevölkerungsmehrheit seit langem akzeptiert - als in der Praxis selten gewählte Alternative zur palliativen Behandlung und zum oft nicht thematisierten, aber leider existierenden einsamen Gewaltsuizid (mit seinen für die Umgebung und Angehörigen oft traumatischen Folgen).

- Drei Viertel der Schweizer Bevölkerung, eine Mehrheit der Ärzteschaft sowie die fünf Schweizer Sterbehilfevereine sehen die Suizidhilfe explizit als Teil der ärztlichen Aufgaben an. Diese gesellschaftliche Realität sollte auch von der SAMW nachvollzogen werden und in deren Richtlinien einfliessen.

- Sterbehilfeorganisationen sind in der Schweiz von der weit überwiegenden Mehrheit akzeptiert. Dies trifft insbesondere für die Organisation EXIT $\mathrm{zu}$, die sich in den mittlerweile 30 Jahren ihres Bestehens zu einer anerkannt seriösen und äusserst transparent kommunizierenden Organisation entwickelt hat, die nach fundierten Abklärungen jedes einzelnen Falles Gewähr bietet, dass in wohlerwogenen und begründeten Fällen ein Suizid unter fürsorglicher Begleitung für alle Involvierten möglichst friedvoll, schmerzlos und sicher sowie ohne traumatische Folgen für Dritte stattfinden kann.

«Die von der ZEK geforderte

\section{breite Diskussion hat längst statt- gefunden.»}

In Berücksichtigung all dieser Tatsachen ist die SAMW gefordert, ihre Richtlinie «Betreuung von Patientinnen und Patienten am Lebensende» zu überdenken. Der EXIT-Vorstand ist bereit zu konstruktivem Dialog. 